\title{
Dual Frequency Measurements of Ocean Forward Scatter with an Ultrawideband Radar
}

\author{
Pete Hansen, Kim Scheff, Eric Mokole and Edmond Tomas \\ Radar Division \\ Naval Research Laboratory \\ Washington, DC 20375
}

\begin{abstract}
This paper presents an experiment description, along with data examples, for ultrawideband radar measurements of low grazing angle forward scatter associated with a test target mounted above a wind disturbed sea surface. The measurement radar used sub-nanosecond transmit pulses ( $0.15 \mathrm{~ns}$ and $0.25 \mathrm{~ns} 3 \mathrm{~dB}$ pulse widths) at center frequencies of 9 and $4 \mathrm{GHz}$ to achieve resolutions finer than $3 \mathrm{~cm}$ in the range dimension. This resolution was sufficient to fully resolve direct and multipath (both single and double bounce) contributions to the signal reflecting from a tower mounted retro reflector. The geometry produced a nominal 3 degree grazing angle for forward scatter. Dual frequency measurements for a wind disturbed ocean surface highlight changes in the magnitude and the location of the scatter components with time.
\end{abstract}

\section{INTRODUCTION}

A knowledge of the behavior of radar forward scatter from the ocean surface is important for designing Navy radar systems and defining their performance. Radar targets physically located close to the ocean surface are often illuminated by radiation coming both directly from the radar and, by reflection, from the ocean surface (see Fig. 1). Interference at the target and on the reciprocal path causes fluctuations in the amplitude of the detected radar signal and distorts the measured phase front.

Simple models for the radar geometry generally describe the combined signal as being formed by components following one direct and three surface bounce paths: (1.) direct from the radar to the target and direct back to the radar; (2.) a single bounce from the radar to the target and direct back to the radar; (3.) direct from the radar to the target and a single bounce back to the radar; (4.) a single bounce from the radar to the target and a single bounce back to the radar. The combined, received signal voltage can be expressed as

$$
\mathrm{E}_{\text {total }}=E\left(\mathrm{t}_{0}\right) \mathrm{G}_{\text {direct }}+2 \mathrm{pE}\left(\mathrm{t}_{1}\right) \mathrm{G}_{\text {single }}+\mathrm{p}^{2} \mathrm{E}\left(\mathrm{t}_{2}\right) \mathrm{G}_{\text {double }}
$$

where the reflection coefficient, $p$, is normally assumed to be the same for all paths. Note that the relative peak powers of the three components are $1.0,4 \mathrm{p}^{2}$ and $\mathrm{p}^{4}$, respectively, for the case where all path gains, $G$, are equal. When a radar at a low elevation illuminates a target which is close to the ocean surface, the geometry tends to include small grazing angles with inherently small differences in both path length (time delay) and direction (angle) between direct and multipath signal components. Experimental measures of the forward scatter are challenging since they require ultra high resolution in time (sub-nanosecond) or angle (fractional degree) or must be inferred from the combined signal [1]. Time resolution, when possible, also presents the advantage of enabling identification and location of multiple scattering points.

To meet this experimental challenge, a specialized, ultrawideband, ultra high resolution, dual polarized measurement radar system [2] has been used in a field experiment to simultaneously measure ocean forward scatter at 9 and $4 \mathrm{Ghz}$ center frequencies. This paper describes the experiment and provides samples of dual polarized forward scatter data.

\section{HARDWARE AND MEASUREMENT GEOMETRY}

A simplified block diagram of the measurement radar is shown in Fig. 2. The field deployable, dual polarized system utilizes two video impulse excited traveling wave tube amplifiers to produce transmit pulses with $2 \mathrm{KW}$ peak power, durations of 0.15 and $0.25 \mathrm{~ns}(3 \mathrm{~dB}$ power envelope) and center frequencies of 9.1 and $3.9 \mathrm{GHz}$, respectively. Fig. 3 shows an oscilloscope recording of the transmit if voltage waveforms and the detected power envelopes. Separate, ultrawideband (2 to $18 \mathrm{GHz}$ response), dual polarized transmit and receive horn antennas are employed in order to maintain receiver isolation without the use of a duplexer. The antennas produce nominal 20 and 45 degree beam widths at the 9 and $4 \mathrm{GHz}$ center frequencies. Polarization ports are selected with low loss diode switches. On receive, the radar system employs a limiter and low noise amplifier followed by an all pass, 90 degree if phase shifter. The receive system detects signals in coherent baseband quadrature form with a unique direct sampling detector based on a multiple sampling head, eight bit digital sampling oscilloscope. The sampling heads simultaneously sample both the rf in-phase and 90 degree phase shifted receive signals. Power envelopes are generated computationally by forming the sum of the squares of the digitally recorded quadrature components.

This detection technique supports an overall instantaneous receive bandwidth in excess of $5 \mathrm{GHz}$. Since only one range point is sampled per pair of sampling heads per pulse, rapid sampling of single or multiple range points is achieved by using repetition rates as high as $100 \mathrm{KHz}$. Either transmit frequency or polarization state (VV, $\mathrm{HH}, \mathrm{VH}, \mathrm{HV})$ can be switched on a pulse-to-pulse basis. Range resolution of the received data is determined by the transmit pulse width and, when using a range scanning mode, by the range sample interval. For the 0.15 ns transmit pulse, basic range. 
resolution is approximately $2 \mathrm{~cm}$ while for the $0.25 \mathrm{~ns}$ pulse the resolution is approximately $3 \mathrm{~cm}$.

The radar system was located at an island site in the Bahamas and was positioned to look out over a wind disturbed ocean surface and illuminate a retro reflector mounted on a tower in the ocean. As diagrammed in Fig. 4, the height of the radar antenna above the ocean surface was $4.3 \mathrm{~m}$, the height of the tower mounted retro reflector was 8.1 $\mathrm{m}$ and the distance from the radar to the retro reflector was $242 \mathrm{~m}$. The retro reflector was mounted on a horizontal bar oriented end-on to the radar and providing approximately $2 \mathrm{~m}$ of range clearance in front of the tower structure. Minimum ocean depth in the tower direction was 10 meters. Fig. 5 shows a view of the radar looking toward the tower.

The calculated grazing angle to the nominal specular reflection point (at a range of 84 meters on the median ocean surface) was 2.9 degrees. With this grazing angle, the known 9.0 GHz reflection coefficients for a flat salt water surface are approximately 1.0 and 0.55 for $\mathrm{HH}$ and $\mathrm{VV}$ polarization states, respectively. Similarly, the reflection coefficients at 4 $\mathrm{GHz}$ are approximately 1.0 and 0.45 . The calculated path length difference between the direct and the single (and between the single and the double) bounce paths for a reflection from this specular point is approximately $30 \mathrm{~cm}$ or $1.0 \mathrm{~ns}$ in received time separation. The retro reflector was a trihedral with a $1.0 \mathrm{~m}$ side dimension and a theoretical monostatic cross section of $960 \mathrm{~m}^{2}$ at $9 \mathrm{GHz}$ and $190 \mathrm{~m}^{2}$ at 4 $\mathrm{GHz}$. A trihedral retro reflector provides a broad monostatic response at the expense of a narrow bistatic response [3]. Consequently, the direct and double bounce paths for this experiment geometry experience a similar path gain while the single bounce path experiences a relative loss of approximately $12 \mathrm{~dB}$ at $9 \mathrm{GHz}$ and $2 \mathrm{~dB}$ at $4 \mathrm{GHz}$.

\section{EXPERIMENTAL MEASUREMENTS}

Measurements of the radar return from the retro reflector were taken over several different days at wind speeds ranging from 6 to 11 knots. The wind conditions were relatively constant with durations of at least 4 hours prior to the measurements. The radar was always positioned to point directly at the retro reflector.

An example of measurements of the return signal from the retro reflector, taken by the system while using a repetitive range profiling mode, is shown in Fig. 6. This data was produced by recording return signals from one range point per pulse over 200 contiguous, $0.3 \mathrm{~cm}$ range spaced $(20 \mathrm{ps}$ time spaced) increments for a total range profile window of $60 \mathrm{~cm}$ (4.0 ns time span). Each range point was sampled for both radar transmit frequencies (with an $\mathrm{HH}$ polarization state) within a time period of 25 microseconds before moving to the next range point and each full range profile was completed within $5.0 \mathrm{~ms}$. Five hundred consecutive range profiles, performed at intervals of $0.3 \mathrm{~s}$ and spanning a total time period of approximately 150 seconds, are shown. Figure axes are time separation in range expressed in nanoseconds, elapsed time in seconds and received peak power on a linear scale.
Fig. 6 displays a typical dual frequency time history of the direct and multipath return signals from the retro reflector for a wind disturbed ocean surface. The wind speed was a steady 11 knots and the orientation was approximately 45 degrees to upwind. In this horizontally polarized data, the relatively steady return signal nearest in range is the direct return from the retro reflector. The second signal in range is the constructive sum of the returns for the two single bounce paths. The third signal in the range window is the return for the double bounce path. In contrast, Fig. 7 shows dual frequency data measured with a relatively calm 6 knot wind. Figures 8 and 9 show 1.5 second samples of the same two data sets in order to illustrate the time varying quality of the scatter between individual records.

Given specular reflections from a flat sea surface, the measured data should exhibit no more than three separate returns, equally spaced in range and with amplitudes controlled by the simple reflection coefficient and gain relation. The actual return data shows evidence of multiple reflection points, conditions where little or no multipath is detected and even focusing effects which modify the gain characteristics. Note that the third, double bounce, signal should not exceed the magnitude of the first, direct signal, unless the effective reflection coefficient for that instant is greater than unity.

Since the direct and double bounce paths should have a relative peak power ratio directly related to the fourth power of the reflection coefficient, a comparison of amplitude distributions for these two distinct return signals should allow a measure of the effective reflection coefficient as a function of radar frequency and sea conditions. Figures 10 through 13 show amplitude distributions for 150 second measurement records. The distributions are plotted on a Weibull amplitude scale with the horizontal axis showing $10 \mathrm{db}$ per division. Figures 10 and 11 examine the dual frequency data records taken for $\mathrm{HH}$ polarization and the two wind speeds. Figures 12 and 13 show dual polarization data (VV and $\mathrm{HH}$ ) for each transmit frequency and with the 6 and $11 \mathrm{knot}$ wind conditions overlaid. Since the distributions are plotted on a decibel scale, the difference between the direct and the double bounce signal divided by four is the $\mathrm{dB}$ level of the reflection coefficient.

\section{DATA OBSERVATIONS}

A comparison of the data sets shows that there is a significant difference in the quality of the forward scatter associated with the radar center frequency. For the relatively undisturbed situation (with 6 knot winds) the forward scatter at $4 \mathrm{GHz}$ is quite steady with a median reflection coefficient close to the flat surface values. In contrast, the forward scatter at $9 \mathrm{GHz}$ shows occasional strong variations and the median reflection coefficient is already somewhat less than that for a flat surface. For the disturbed situation (with 11 knot winds) the forward scatter at $4 \mathrm{GHz}$ exhibits significant variations, even though the median reflection coefficients have not decreased significantly. It should be noted that the 4 $\mathrm{GHz}$ scatter for this disturbed ocean is exhibiting a significant 
number of instances with a positive gain (reflection coefficient greater than 1.0 for $\mathrm{HH}$ ). At $9 \mathrm{GHz}$, the disturbed surface causes the median reflection coefficients to decrease and increases the range of variations.

While the flat surface reflection coefficients for the two center frequencies are quite similar, a calculation of the extent of the first Fresnel zone for this experiment geometry shows that the $4 \mathrm{GHz}$ reflection does take advantage of a specular region which contains approximately three times the area of that contained in the $9 \mathrm{GHz}$ region. This difference may partially account for the relatively steady reflections measured for the lower frequency situation.

It is apparent that the disturbed surface is causing a wide range of instantaneous reflection values for both center frequencies. When there is a strong variation in the scatter, the rate of change appears to be significantly greater for 9 $\mathrm{GHz}$ than for $4 \mathrm{GHz}$. This may simply be a result of sensitivity at the shorter wavelength to physical movement. The general variations for both transmit frequencies, as expected, appear to be tied to the relatively slow motion of the large scale ocean features.

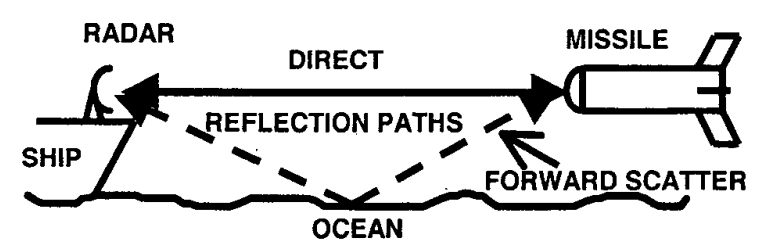

Fig.1 Situation for low grazing angle forward scatter.
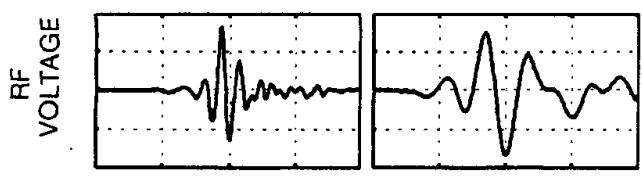

$9 \mathrm{GHz}$ $4 \mathrm{GHz}$

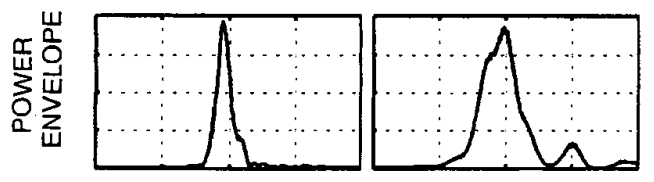

TIME (0.4 NANOSECONDS/DIV)

Fig. 3 Transmit waveforms.

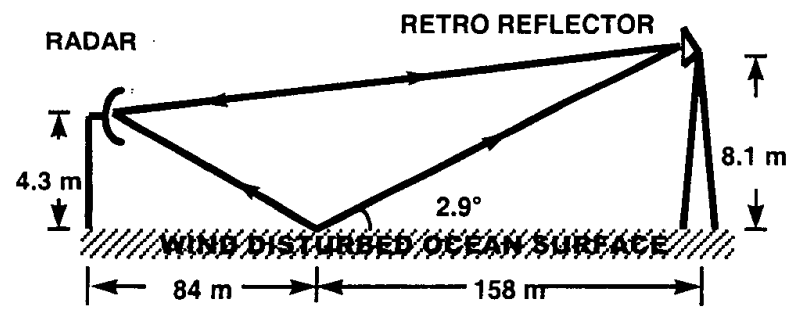

Fig. 4 Experiment geometry.

\section{CONCLUSIONS}

An ocean forward scatter experiment has been described which utilizes an ultrawideband, dual polarized, dual frequency instrumentation radar. The better than $3 \mathrm{~cm}$ range resolution of the radar system has served to separate the direct and multipath components of signals reflected from targets oriented close to the ocean surface. The ability to achieve this separation and examine the signal components in isolation is leading to a better understanding of the composition and behavior of low grazing angle forward scatter. These initial experimental results have emphasized differences in the forward scatter associated with radar center frequency as a function of wind and sea conditions.

\section{REFERENCES}

[1] P. Beckmann and A. Spizzichino, The Scattering of Electromagnetic Waves from Rough Surfaces. New York: Macmillan Company, 1963, ch.13, pp. 292-305.

[2] P. Hansen and K. Scheff, "Ultrawideband sea clutter, system and measurements at X-Band," in Proceedings of the IEEE 1998 National Radar Conference, 1998, pp. 393-398.

[3] C. G. Bachman, Radar Targets. Toronto: D.C. Heath and Company, 1982, ch.2, pp. $79-80$

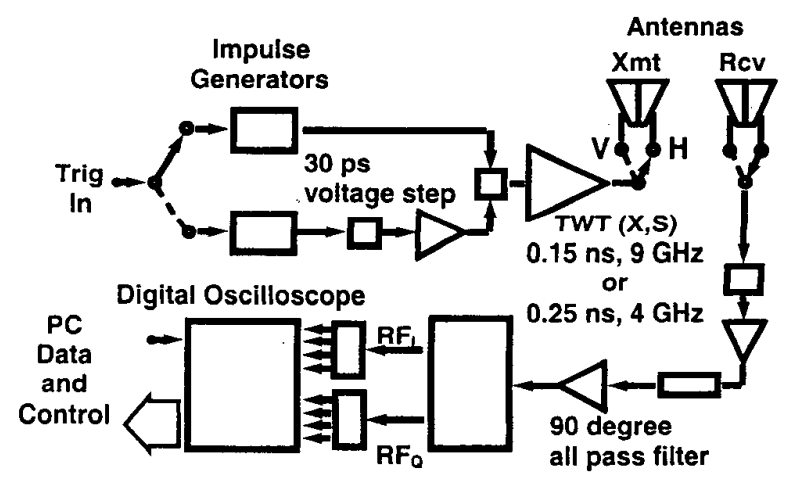

Fig. 2 Block diagram of radar

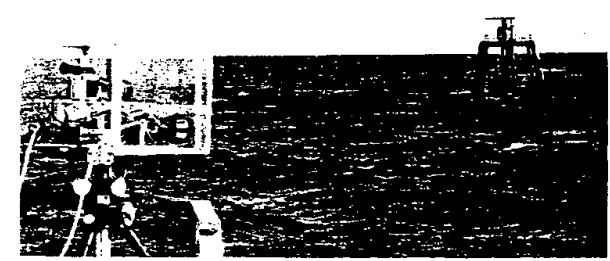

Fig. 5 View of radar looking toward tower. 


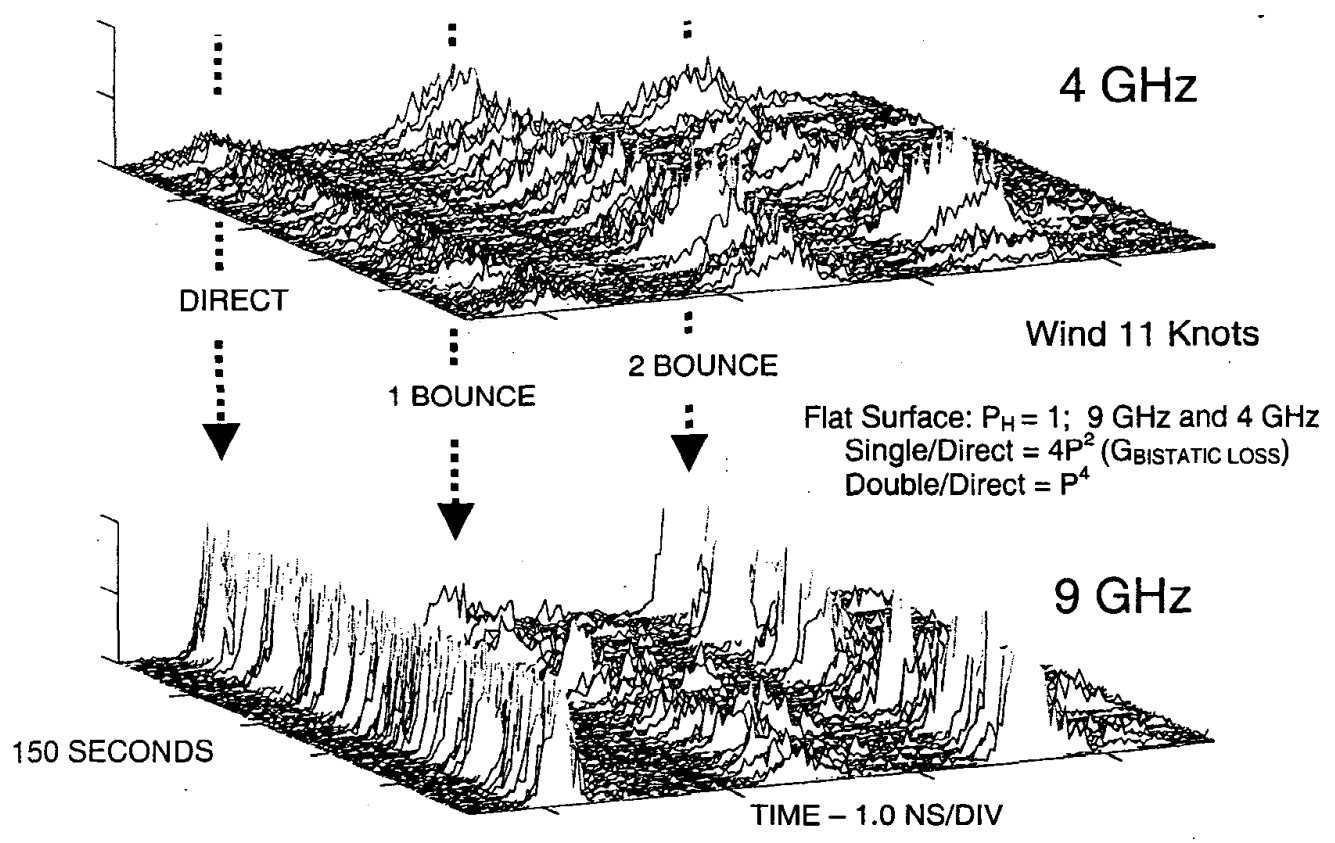

Fig. 6 Dual frequency multipath returns, 11 knot winds, HH polarization.

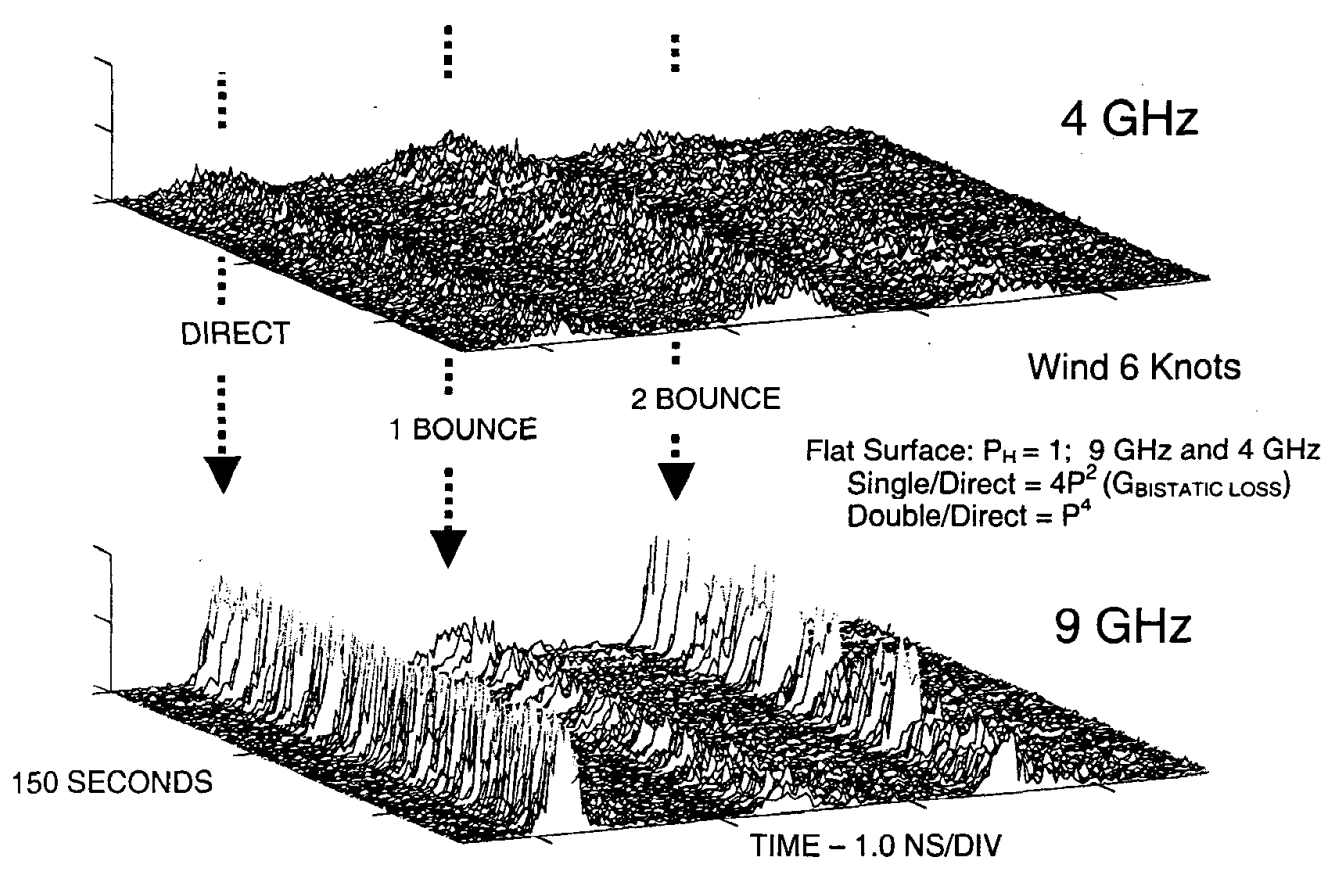

Fig. 7 Dual frequency multipath returns, 6 knot winds, $\mathrm{HH}$ polarization. 


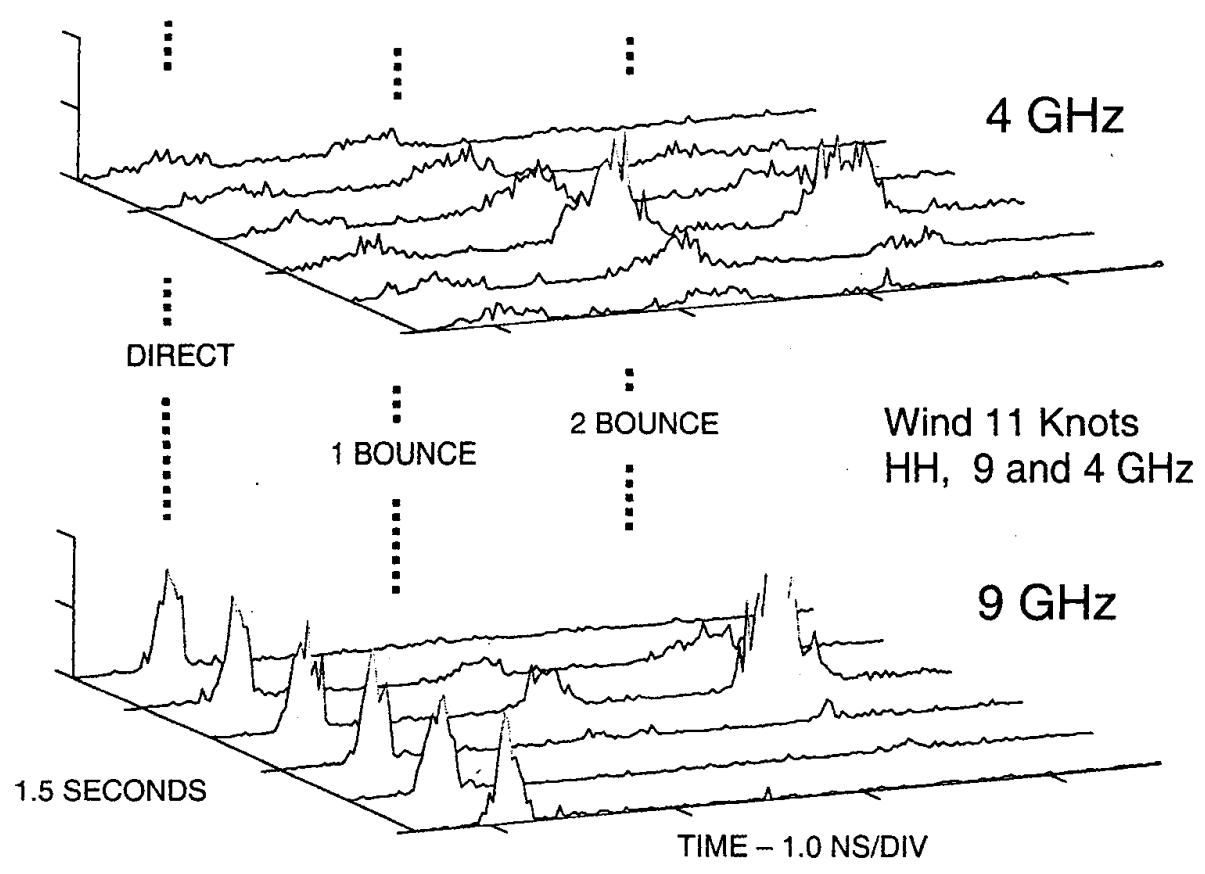

Fig. 8 Short record of dual frequency multipath returns, 11 knot winds.

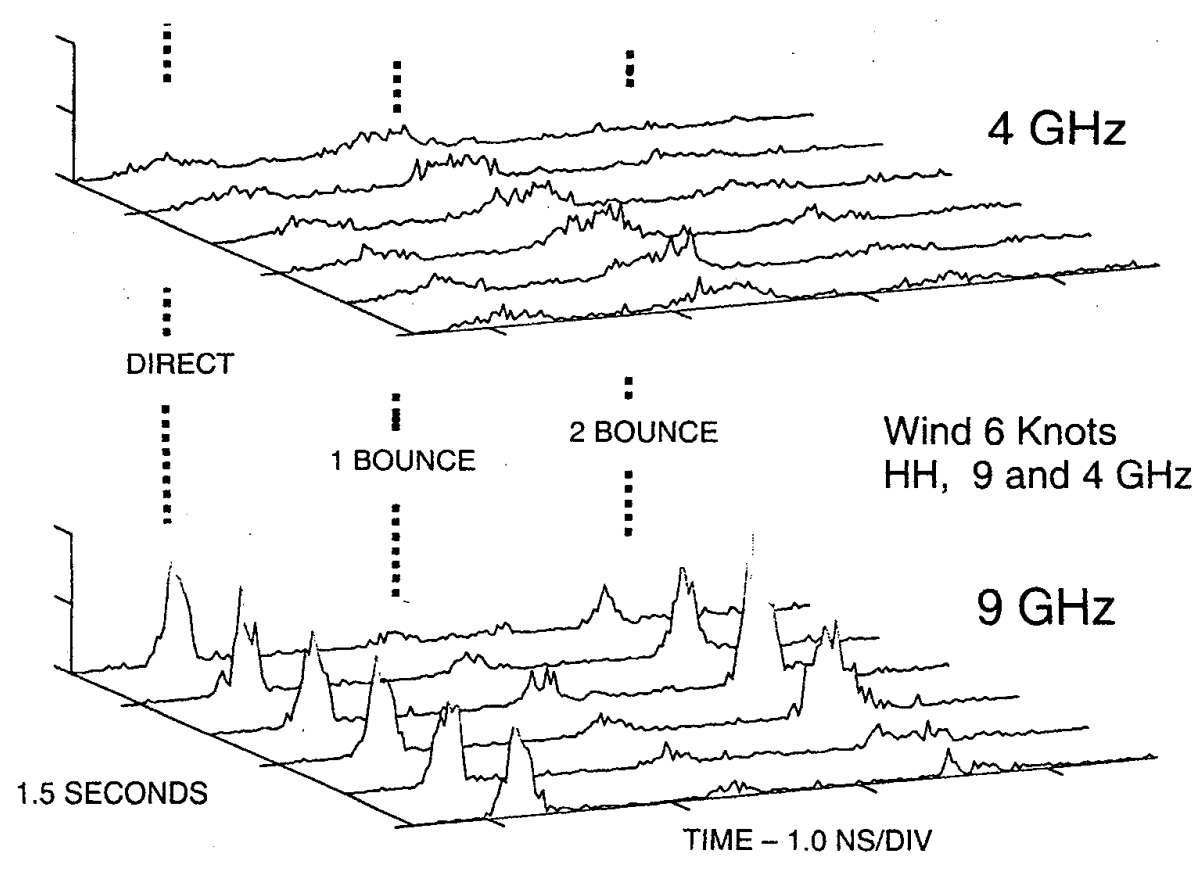

Fig. 9 Short record of dual frequency multipath returns, 6 knot winds. 


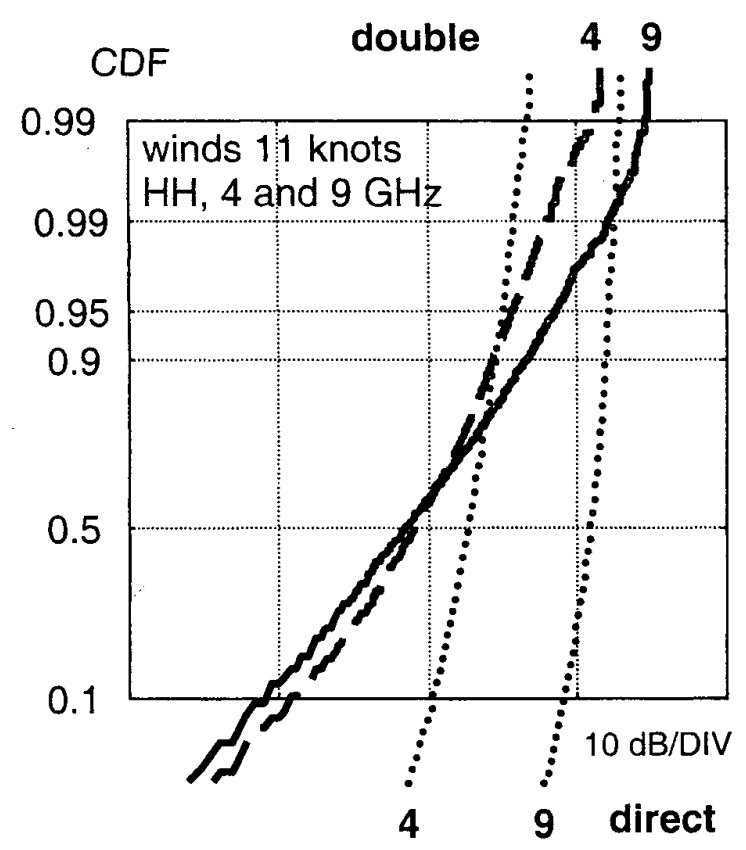

Fig. 10 Cumulative distributions, dual frequency, 11 knot winds.

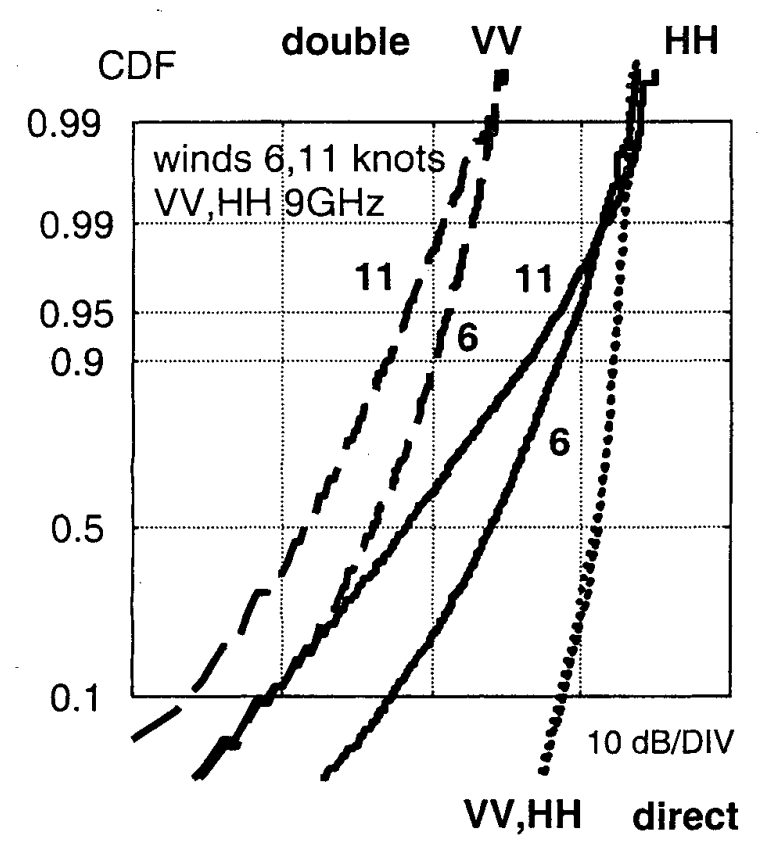

Fig. 12 Cumulative distributions, $9 \mathrm{GHz}$, dual polarization, 6 and 11 knot winds.

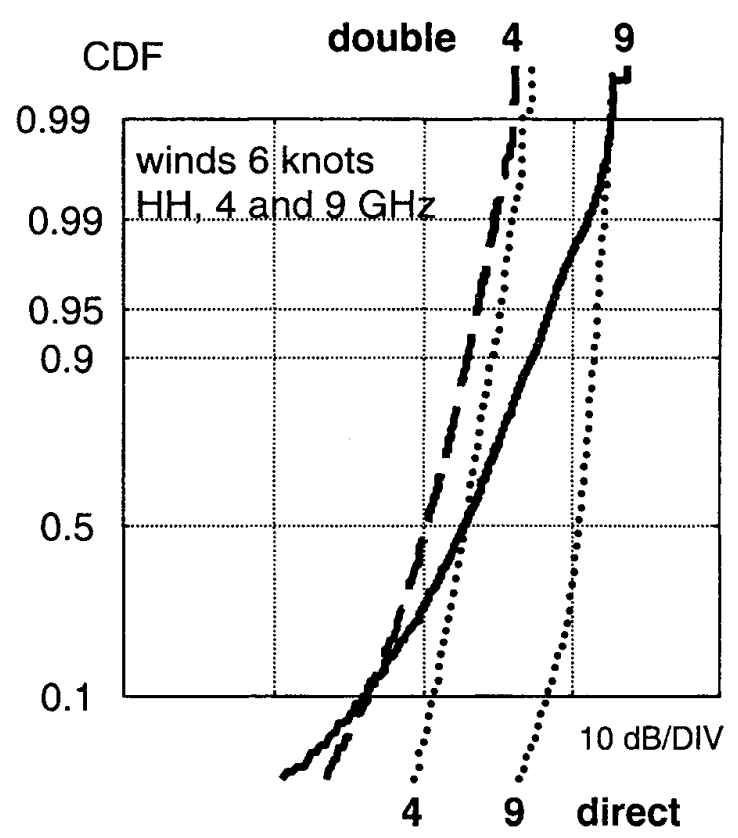

Fig. 11 Cumulative distributions, dual frequency, 6 knot winds.

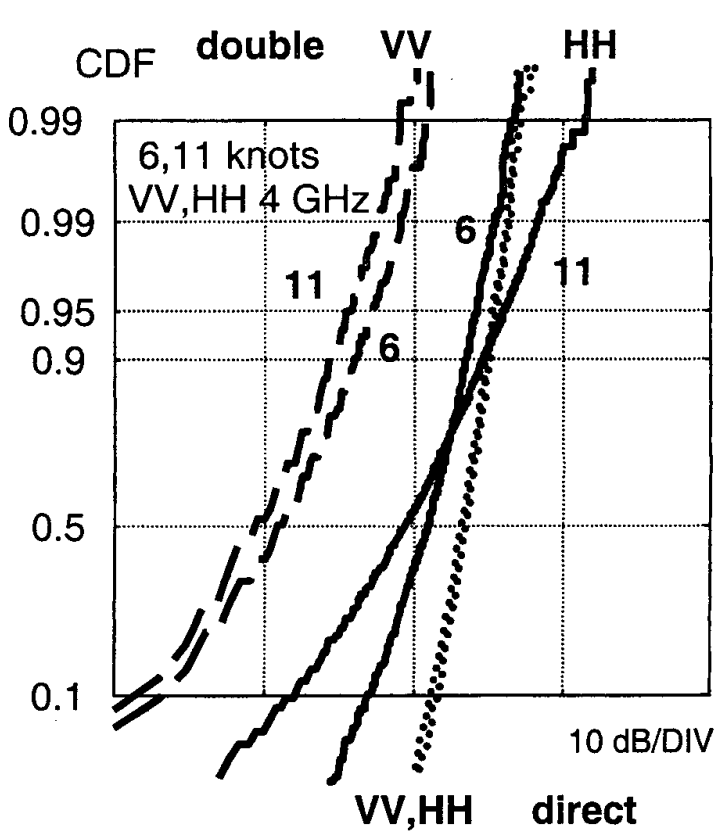

Fig. 13 Cumulative distributions, $4 \mathrm{GHz}$, dual polarization, 6 and 11 knot winds. 